\title{
Critical Behaviour of the Fuzzy sphere
}

\author{
Denjoe O'Connor ${ }^{1}$, Brian P. Dolan ${ }^{1,2}$, and Martin Vachovski ${ }^{1}$ \\ ${ }^{1}$ School of Theoretical Physics, \\ Dublin Institute For Advanced Studies, \\ 10 Burlington Rd, Dublin 4, Ireland; \\ ${ }^{2}$ Department of Mathematical Physics, \\ National University of Ireland Maynooth, Maynooth, Ireland
}

August 30, 2013

\begin{abstract}
We study a multi-matrix model whose low temperature phase is a fuzzy sphere that undergoes an evaporation transition as the temperature is increased. We investigate finite size scaling of the system as the limiting temperature of stability of the fuzzy sphere phase is approached. We find on theoretical grounds that the system should obey scaling with specific heat exponent $\alpha=\frac{1}{2}$, shift exponent $\bar{\lambda}=\frac{4}{3}$ and that the peak in the specific heat grows with exponent $\bar{\omega}=\frac{2}{3}$. Using hybrid Monte Carlo simulations we find good collapse of specific heat data consistent with a scaling ansatz which give our best estimates for the scaling exponents as $\alpha=0.50 \pm 0.01, \bar{\lambda}=1.41 \pm 0.08$ and $\bar{\omega}=0.66 \pm 0.08$.
\end{abstract}

\section{Introduction}

Different approaches to the basic structure of spacetime exist and in recent years there has been a growing interest in the notion of space or spacetime as an emergent concept. A natural setting where space or spacetime are necessarily emergent is in the proposed nonpterturbative definitions of string theory provided by matrix models [1, 2, 3. In this context plausible models of emergent geometry have been discussed 4 . The notion of classical geometry changes drastically within the context of matrix models; neither background geometry nor topology is predefined but instead they emerge dynamically as a consequence of the condensation of the matrix degrees of freedom to form the background geometry.

The purpose of this paper is to study the scaling of finite matrix effects for large matrix size (large $N$ ) as the limit of stability of the geometry is approached.

We consider an action in which the basic objects are simple Hermitian matrices at finite temperature with a prescribed energy functional. The geometry exists at low temperatures as a condensate around which the system fluctuates.

The energy functional of interest here is that of a 3-matrix model consisting of the trace of the square of the commutator of the matrices (a Yang-Mills term) plus the epsilon-tensor contracted with the trace of the three matrices (a Myers 
term [5]). The system has been studied before in 6, 7, 8, 9, 10, 11 and with a mass deformation in 12. It is a static bosonic subsector of the BMN model 3 .

The model exhibits a geometrical phase for sufficiently low temperatures where the effect of the cubic Myers term is important, with the geometry being that of a fuzzy sphere [13, 14] which is a non-commutative version of the commutative sphere.

At a critical temperature, which can be traded for a critical Myers coupling, a phase transition occurs and the condensed geometry evaporates. In the geometrical, low temperature phase, small fluctuations around this condensate correspond to a $U(1)$ gauge and scalar field multiplet $[7$.

In previous studies [10, 11] it was argued that, as the transition is approached from the low temperature side, there are divergent fluctuations in the system and in particular that the specific heat diverges with exponent $\alpha=\frac{1}{2}$. This suggests that the system may exhibit finite size scaling [15, 16] in terms of the matrix size as the system size grows. We therefore study the growth of fluctuations, in terms of both temperature and matrix size, as the transition is approached from below, but insisting on remaining in the fuzzy sphere phase. The fluctuations we consider are those of a restricted ensemble and do not take into account the rare finite matrix transitions when the system jumps from the fuzzy sphere to the matrix phase. Such fluctuations are completely absent in the large $N$ limit as is typical of matrix models where tunneling goes to zero as $N$ goes to infinity. Our study shows that the fluctuations do indeed scale with matrix size.

The principal results of this paper are:

- In the absence of fluctuations, the Myer's term gives rise to an instability of the model. This is responsible for the destabilisation of the matrix phase for small matrices but insufficient to cause an instability for matrices of size $N \geq 12$ approximately.

- The transition is rounded by finite matrix effects and there is a pseudo critical temperature shifted from the infinite $N$ transition temperature with shift exponent which we predict on scaling grounds is $\bar{\lambda}=\frac{4}{3}$.

- The scaling form of the free energy with $N$ is a universal function of $x=t N^{\bar{\lambda}}$ where $t=\frac{T_{c}-T}{T_{c}}$. We predict the scaling relation $\bar{\lambda}=\frac{2}{2-\alpha}$.

- If we assume that $d=2$, based on a fuzzy sphere background, and $\alpha=\frac{1}{2}$ as found in earlier studies, then our theoretical prediction is that $\bar{\lambda}=\frac{4}{3}$ and if further we accept that the approach to criticality is governed by a divergent correlation length then we infer that the correlation length exponent $\nu=\frac{2}{3}$.

- Hybrid Monte Carlo simulations give good agreement with finite size scaling in terms of the matrix size $N$ and assuming the asymptotic scaling form of the specific heat as the critical temperature is approached from below $C_{v} \simeq A_{-}\left(T_{c}-T\right)^{-\alpha}$ our best estimates from these measurements are $A_{-}=0.051 \pm 0.017, \alpha=0.50 \pm 0.01, \bar{\lambda}=1.41 \pm 0.08$ and $\bar{\omega}=0.66 \pm 0.08$ where the peak in the specific heat grows as $A_{\bar{\omega}} N^{\bar{\omega}}$. These are in good agreement with the theoretical estimates based on scaling and $\alpha=\frac{1}{2}$. 
- When we assume the exponent $\alpha=\frac{1}{2}$ and scaling so that $\bar{\lambda}=\frac{4}{3}$ and $\bar{\omega}=\frac{2}{3}$ we find tight estimates for the shift amplitude $A_{\bar{\lambda}}=3.9 \pm 0.1$ and the specific heat maximum amplitude $A_{\bar{\omega}}=0.199 \pm 0.005$.

The organization of the paper is as follows: In section $\S 2$ we discuss the main properties of the model which are relevant to us. In section $\S 3$ we present the theory of finite size scaling. In section $\S 4$ we give an overview of the difficulties that arise in near-critical system simulations and the impact they have on our studies. In section $\S 5$ we present our numerical results. In section $\S 6$ we present our conclusions from the study.

\section{The three matrix model}

The model we shall consider in this paper is the three matrix model, which was studied in 6, 9, 10, 11. Let $X_{a}, a=1,2,3$, be three traceless $N$-dimensional Hermitian matrices. We consider the action (really an energy divided by temperature, as all our considerations will be in Euclidean signature)

$$
\mathcal{S}[X]=N \operatorname{Tr}\left(-\frac{1}{4}\left[X_{a}, X_{b}\right]^{2}+\frac{2 i g}{3} \epsilon_{a b c} X_{a} X_{b} X_{c}\right)
$$

where $\epsilon_{a b c}$ is the totally antisymmetric Levi-Civita symbol, $g \in \mathbb{R}$ is a parameter of the model. The change $g \rightarrow-g$ is equivalent to $X_{a} \rightarrow-X_{a}$, therefore it will be sufficient to restrict our study to the case $g \geq 0$, which we shall assume. This model has a phase transition [6, 9, and it is the vicinity of this transition that is of interest to us here.

The stationary points of the system follow from varying $\mathcal{S}$ : demanding that $\delta \mathcal{S}=0$ results in

$$
\left[X_{b},\left[X_{a}, X_{b}\right]-i g \epsilon_{a b c} X_{c}\right]=0,
$$

and every configuration of matrices $X_{a}$ that solves (2) is a (local) extremum or saddle point of (1).

For most purposes of this paper it will be convenient to scale out a factor of $\sqrt{N}$ and work with the parametrization $\widetilde{g}=g \sqrt{N}$, as this gives a phase diagram that does not dependent on $N$. So we make the substitution $X_{a} \rightarrow \widetilde{g} \frac{D_{a}}{\sqrt{N}}$ in terms of which the action reads

$$
\mathcal{S}[D]=\frac{\widetilde{g}^{4}}{N} \operatorname{Tr}\left(-\frac{1}{4}\left[D_{a}, D_{b}\right]^{2}+\frac{2 i}{3} \epsilon_{a b c} D_{a} D_{b} D_{c}\right) .
$$

From this it is clear that observables are symmetric under $\widetilde{g} \rightarrow-\widetilde{g}$, or equivalently under $g \rightarrow-g$. For $\widetilde{g} \neq 0$ we can interpret $T=\widetilde{g}^{-4}$ as a temperature for the system 1

Many of the physical properties are characterised by the expectation value of the action $\langle\mathcal{S}\rangle$, the specific heat of the system and the distribution of the eigenvalues of the matrices $X_{a}$. Our study here will focus on the first two. The specific heat is defined as

$$
C_{v}=\frac{\left\langle(\mathcal{S}-<\mathcal{S}>)^{2}\right\rangle}{N^{2}}=\frac{\langle\mathcal{S}\rangle}{N^{2}}-\widetilde{g}^{4} \frac{d}{d \widetilde{g}^{4}}\left(\frac{<\mathcal{S}>}{N^{2}}\right) .
$$

\footnotetext{
${ }^{1}$ Generally we prefer to discuss the physical properties of the system in terms of the temperature $T$, though it may be more convenient in some situations to use either $\widetilde{g}$ or $g$. Note that, as there are no dimensionful quantities in the action, $T$ here is dimensionless.
} 
For convenience we shall define $S:=<\mathcal{S}\rangle$, this is the internal energy divided by the temperature.

The model has at least two phases, which we call the commuting matrix phase and the fuzzy sphere phase, and the above three quantities behave quite differently in these two phases. In a semi-classical approximation [10] the phase transition occurs at

$$
\widetilde{g}_{c}=\left(\frac{8}{3}\right)^{\frac{3}{4}} \Leftrightarrow T_{c}=\left(\frac{3}{8}\right)^{3} \approx 0.05273 .
$$

This is remarkably close to our numerical result of $0.0531 \pm 0.0003$ obtained in $\$ 5.1$.

\subsection{The commuting matrix phase}

The high temperature phase of the model with $T>T_{c}$ is characterised by fluctuations around a ground state in which the three matrices are mutually commuting. This ground state can be represented by $X_{a}$ 's which are linear combinations of $h H_{m} h^{-1}, m=1, \ldots, N-1$, where $H_{m}$ are in the Cartan subalgebra of $s u(N)$ and $h \in S U(N)$. Any such linear combination is a trivial solution to (21), so the classical action vanishes for these stationary configurations.

However, these solutions can be unstable if any of the eigenvalues get too close to one another, as we now demonstrate. Fluctuations around a classical solution can be expressed as

$$
X_{a}=X_{0, a}+\delta X_{a},
$$

with $X_{0, a}$ three mutually commuting, Hermitian matrices. We are free to perform an $N \times N$ unitary transformation on $X_{0, a}$ to simultaneously diagonalise them,

$$
\left(X_{0, a}\right)_{i j}=\lambda_{i}^{a} \delta_{i j}, \quad(\text { no sum over } i)
$$

A little algebra reveals that, to quadratic order in $\delta X_{a}$ we have

$$
-\frac{1}{4} \operatorname{Tr}\left[X_{a}, X_{b}\right]^{2}=\frac{1}{2}\left(\left(\Delta_{i j} \cdot \Delta_{i j}\right) \delta^{a b}-\Delta_{i j}^{a} \Delta_{i j}^{b}\right) \delta X_{a, i j} \delta X_{b, i j}
$$

and

$$
\frac{2 i}{3} \epsilon_{a b c} X_{a} X_{b} X_{c}=i \epsilon_{a b c} \Delta_{i j}^{c} \delta X_{a, i j} \delta X_{b, i j},
$$

where $\Delta_{i j}^{a}=\lambda_{i}^{a}-\lambda_{i}^{a}$. Stability of fluctuations around a classical solution are therefore determined by the eigenvalues of the operator

$$
\frac{1}{2}\left(\Delta_{i j}^{2} \delta^{a b}-\Delta_{i j}^{a} \Delta_{i j}^{b}\right)+i g \epsilon_{a b c} \Delta_{i j}^{c}
$$

where $\Delta_{i j}^{2}=\Delta_{i j}^{a} \Delta_{i j}^{a}$ with eigenvalues $0, \frac{1}{2} \Delta_{i j}^{2} \pm g \sqrt{\Delta_{i j}^{2}}$. The zero-eigenvalue is associated with the $U(N)$ invariance of the action and can be removed by gauge fixing such that one of the matrices is diagonal. However, if some of the background eigenvalues, $\lambda_{i}^{a}$, are too close together, (10) has a negative eigenvalue, and hence an instability. In particular, if

$$
\Delta_{i j}^{2}<4 g^{2}
$$


for any pair $i, j$, then there is a direction which is unstable. The solution is stable if all the eigenvalues of $X_{0, a}$ are far enough apart. Note that there are no unstable directions for $g=0$, the instability is induced by the Myers' term.

Fluctuations can of course modify this analysis. It is possible that they stabilise the unstable solutions. We will not attempt an analytic approach to this question here but will return to it later in the paper. The first immediate effect of fluctuations is that they modify the expectation value of the action and shift it away from $S=0$. To study this effect consider a Schwinger-Dyson type analysis,

$$
\begin{aligned}
0 & =\int[D X] \operatorname{Tr} \frac{\partial}{\partial X_{a}}\left(X_{a} e^{-\mathcal{S}}\right) \\
\Rightarrow \quad 0 & =3\left(N^{2}-1\right)-\operatorname{Tr}<X_{a} \frac{\partial \mathcal{S}}{\partial X_{a}}> \\
\Rightarrow \quad 3\left(N^{2}-1\right) & =-N T r<\left[X_{a}, X_{b}\right]^{2}>+2 i g N \epsilon_{a b c} \operatorname{Tr}<X_{a} X_{b} X_{c}> \\
& =4<\mathcal{S}>-2 i g N \epsilon_{a b c} \operatorname{Tr}<X_{a} X_{b} X_{c}>,
\end{aligned}
$$

where $3\left(N^{2}-1\right)$ is the number of degrees of freedom in the three Hermitian matrices $X_{a}$. Thus we expect

$$
\frac{<\mathcal{S}>}{N^{2}}=\frac{3\left(N^{2}-1\right)}{4 N^{2}}+\frac{i g}{2 N} \epsilon_{a b c} T r<X_{a} X_{b} X_{c}>.
$$

It is shown numerically in [12] that $\operatorname{Tr}<X_{a} X_{b} X_{c}>\approx \frac{1}{N^{1 / 2}}\left(\frac{1}{T^{1 / 4}}+o\left(\frac{1}{T^{1 / 2}}\right)\right)$ at large $T$ and large $N$ so, in this limit,

$$
\frac{S_{m}(T)}{N^{2}}=\frac{3}{4}
$$

which is positive. This suggests that, in the matrix phase of the model, the specific heat does not depend on $T$,

$$
C_{v}=\frac{3}{4}
$$

and each degree of freedom contributes a value of $\frac{1}{4}$ to the specific heat. The model behaves like a pure Yang-Mills matrix model in the large $N$ limit, i.e. one with only the commutator squared term.

For large $N$, the eigenvalue distribution in this phase is compatible with a parabolic distribution [17, 12, 18. In a gauge in which $X_{3}$ is diagonal (which can always be achieved by an $S U(N)$ transformation) the diagonal entries of $<X_{3}>$ can be arranged in descending order and give a parabolic distribution with normalised density

$$
\rho(\lambda)=\frac{3\left(R^{2}-\lambda^{2}\right)}{2 \pi R^{3}}
$$

with $R$ determined numerically to be 2.0. This parabolic form of the distribution further implies, as argued in 18, 19, that the background of commuting matrices have their eigenvalues distributed uniformly within a ball of radius $R=2.0$. Fluctuations around this background are still present and those of the different matrices do not commute. 


\subsection{The fuzzy sphere phase}

The fuzzy sphere phase is a cold (ordered) phase and is radically different to the commuting matrix phase. The background matrices in this phase are represented by a solution to (2) in which $X_{a}$ are proportional the generators of $s u(2), X_{a}=g L_{a}$ with $\left[L_{a}, L_{b}\right]=i \epsilon_{a b c} L_{c}$, up to $U(N)$ transformation $h L_{a} h^{-1}$ with $h \in S U(N)$. For the classical solution we have $\sum_{a} X_{a}^{2}=g^{2} c_{2} \mathbf{1}$, with $c_{2}$ the second order Casimir for the $N$-dimensional representation of $S U(2)$, and hence $\frac{\left\langle\operatorname{Tr} D_{a}^{2}>\right.}{N c_{2}}=1$. More generally we shall define a radius of the fuzzy sphere, $\mathcal{R}$, by

$$
\mathcal{R}^{2}=\frac{<\operatorname{Tr} D_{a}^{2}>}{N c_{2}}
$$

which, in the large $N$ limit, has a nonzero value only in the fuzzy sphere phase. The parameter $\mathcal{R}$ provides an order parameter for the transition, being non-zero in the low temperature phase and zero in the high temperature phase.

For low temperatures below the transition the expectation value of the action in this phase is approximated by the value of the action for the solution $X_{a}=$ $g L_{a}$, so that $\mathcal{R} \simeq 1$ and

$$
S_{f}(T)=-\frac{c_{2} c_{2}^{a d j}}{12 T}+<\text { fluctuations }>
$$

where $c_{2}=\frac{N^{2}-1}{4}$ and $c_{2}^{a d j}=2$ are the Casimir and adjoint Casimir operators of $s u(2)$. Since the matrices $X_{a}$ are proportional to the generators they have a discrete eigenvalue spectrum with $N$ distinct eigenvalues of the form $\lambda=$ $\left\{-g \frac{N-1}{2},-g \frac{N-3}{2} \ldots g \frac{N-1}{2}\right\}$.

\subsection{Excited fuzzy sphere states}

A closer examination of equation (2) shows that there are reducible fuzzy sphere solutions, with $X_{a}$ proportional to $s u(2)$ generators in a reducible representation of the form

$$
R_{1}\left(M_{1}\right) \oplus R_{2}\left(M_{2}\right) \ldots \oplus R_{K}\left(M_{k}\right)
$$

where $R_{i}\left(M_{i}\right)$ is an $s u(2)$ irreducible representation of dimension $M_{i}$ and $\sum_{i=1, K} M_{i}=N$. The matrices $X_{a}$ for this solution can always be chosen to have block-diagonal form and this will be implicit for the rest of this discussion. All of the metastable states with the n-tuple $\left(M_{1}, M_{2}, \ldots, M_{K}\right)$ can be listed and indeed all the solutions described so far - even the commuting matrix phase and the irreducible fuzzy sphere phase - can be classified this way. For a fixed $N$, the number of distinct solutions to (2) of the form (19) grows as $p(N)$, the number of integer partitions of $N$, which for large $N$ behaves as

$$
p(N) \simeq \frac{e^{\pi \sqrt{2 N / 3}}}{4 N \sqrt{3}} .
$$

and for low enough temperatures the fuzzy sphere configuration represents the ground state of the system with each of the other $p(N)-1$ configurations representing a potential metastable state.

The special case $M_{1}=M_{2}=\ldots=M_{N}=1$ corresponds to the commuting matrix phase and the ground state in this case can be viewed as arising from $N$ 
one-dimensional (or trivial) representations of $s u(2)$. In the other extreme, when $K=1$, the representation is irreducible and gives the fuzzy sphere discussed above. Nevertheless, the commuting matrix solution is genuinely different to all the others, the configuration represented by diagonal matrices is a state built from the one-dimensional representations so the $\epsilon$ term in (2) plays no rôle and the system has no memory of $s u(2)$. These are one dimensional representations of an arbitrary algebra.

There are two observables that could be used to distinguish between the fuzzy sphere from section $\$ 2.2$ and these excited configurations with $K>1$. First we can use the eigenvalues of the matrices: since any irreducible representation $R(M)$ of $s u(2)$ has $M$ distinct eigenvalues, configurations of the form (19) have $\max \left\{M_{i}\right\}<N$ distinct eigenvalues in their spectra. Another observable that is sensitive to the excited states is the expectation value of the action. We have

$$
S_{e f}\left(T,\left(M_{1}, M_{2}, \ldots, M_{K}\right)\right)=-\sum_{i=1}^{K} \frac{M_{i} c_{2}\left(M_{i}\right) c_{2}^{a d j}}{12 N T}+<\text { fluctuations }>
$$

and $S_{f}(T)<S_{e f}(T, K)<S_{m}(T)$ for $\forall K: 1<K<N$. These excited states are unstable, see section 4.2 and far from the phase transition fluctuations around the lowest excited states are very much like fluctuations around the ground state (see Fig. 5) and these fluctuations are small relative to the spacing between such states. So at very low temperatures these excited states do not play an important rôle in the thermodynamics of the system.

The considerations so far have been in the absence of fluctuations and apply, at low temperatures, sufficiently far from the critical point that fluctuations can be neglected. Fluctuations are important in the high temperature phase due to eigenvalue repulsion which lifts the degeneracy of eigenvalues and stabilises the high temperature phase against the Myers instability discussed in section \$2.1. Otherwise at high temperatures fluctuations are not large. In the low temperature phase eigenvalue repulsion also lifts the degeneracy associated with identical $s u(2)$ blocks in the low temperature phase otherwise for all practical purposes the excited fuzzy states play no rôle for sufficiently low temperatures.

However, near the critical point as the transition is approached from the low temperature side fluctuations grow and the specific heat rises, see Fig. 3. In this regime the excited fuzzy sphere states will of necessity play a more important rôle. One can estimate when the first excited state will be important by noting that it corresponds to $R(N-1) \oplus R(1)$ and that the difference in action between this and the ground state grows linearly with $N$, being $\frac{N}{8}$ for large $N$. If the square of this difference divided by $N$ is larger than the specific heat then these excited states are unimportant, however, as the critical point is reached the specific heat grows and eventually all excited states are important. Far from the transition $C_{v}=1$, so we can estimate that the first excited state will begin to become important for $T \sim \frac{1}{64} \simeq 0.0156$. Once the first excited state becomes important, there are more possibilities for fluctuations in the system and the specific heat in turn grows. More excited states enter the picture and eventually the system undergoes a phase transition. Earlier estimates 6, 7, 8, 9, 10, give this transition at $T_{c}=\left(\frac{3}{8}\right)^{3} \simeq 0.0527$. So we expect the critical regime between for $0.0156 \leq T \leq 0.0527$ which is quite consistent with Fig. 3 . 


\subsection{A 1.5 order phase transition}

It is clear from the previous sections that the commuting matrix and the fuzzy sphere phases are quite different. The classical results in equations (14) and (18) give $S_{m}\left(T_{c}\right)-S_{f}\left(T_{c}\right) \neq 0$ at the transition temperature, $T_{c}$, so this might naïvely be classified as a first order phase transition - with latent heat and a finite specific heat on either side of the transition - but the full story is more subtle.

An approximate analytic expression for the specific heat, in the $N \rightarrow \infty$ limit, was given in [11. If we make the ansatz $X_{a}=\phi g L_{a}$ in the fuzzy sphere phase and write an effective potential for the theory in terms of $\phi$ then, in a large $N$ semi-classical approximation, equations (3.25) and (3.26) of reference [11] with $m=0$, give, $\mathcal{R}=\phi$ and in the large $\widetilde{g}$ limit,

$$
C_{v}=\frac{3}{4}+\frac{\widetilde{g}^{5} \phi^{2}}{32} \frac{d \phi}{d \widetilde{g}} \quad \text { with } \quad \phi=1-\frac{2}{\widetilde{g}^{4}}-\frac{12}{\widetilde{g}^{8}}+o\left(\frac{1}{\widetilde{g}^{12}}\right)
$$

Thus

$$
C_{v}=1+\frac{2}{\widetilde{g}^{4}}+o\left(\frac{1}{\widetilde{g}^{8}}\right)
$$

and

$$
C_{v} \underset{T \rightarrow 0}{\longrightarrow} 1
$$

On the other hand, near $\widetilde{g}_{c}$

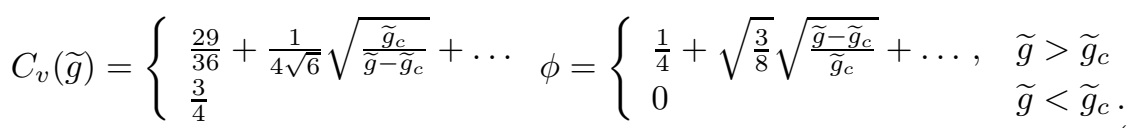

Thus the specific heat diverges 2 on the low temperature side of the transition. This is the characteristic behaviour of a continuous (also called 2nd order) transition near a critical point.

The general theory of continuous phase transitions and critical phenomena [20, 21, 22, 23. suggests that, near the phase transition, the specific heat $C_{v}$ on either side of the transition should behave as

$$
C_{v}(T) \sim C_{0 \pm}+A_{ \pm}\left|T-T_{c}\right|^{-\alpha}
$$

The three matrix model under consideration here seems to have a rather unusual phase transition in that the specific heat diverges only as the phase transition is approached from one side but does not diverge as it is approached from the other.

The internal energy per degree of freedom, $U=T \frac{<\mathcal{S}>}{N^{2}}$, arising from the semi-classical approximation of [11, is plotted in Fig. 1] and the slope of this curve near $T_{c}$, when expressed in terms of $\widetilde{g}$, results in the form (25) for the specific heat. The semi-classical approximation on the low-temperature side is given by

$$
\frac{S}{N^{2}}=\frac{3}{4}-\frac{\phi^{3}(T)}{24 T}
$$

${ }^{2}$ In terms of temperature (25) gives $C_{v}(T)=\frac{29}{36}+\frac{3}{64}\left(T_{c}-T\right)^{-\frac{1}{2}}+\ldots$ 
where

$$
\begin{gathered}
\phi(T)=\frac{1}{4}\left(1+\sqrt{1+\delta(T)}+\sqrt{2-\delta(T)+\frac{2}{\sqrt{1+\delta(T)}}}\right) \\
\delta(T)=4 T^{1 / 3}\left(\left(1+\sqrt{1-\frac{T}{T_{c}}}\right)^{\frac{1}{3}}+\left(1-\sqrt{1-\frac{T}{T_{c}}}\right)^{\frac{1}{3}}\right) .
\end{gathered}
$$

This is the typical behaviour of a critical point and a second order phase transition. This implies that a small correction to $T_{c}$ can give a very large correction to the internal energy, $\Delta U_{T_{c}}$.

On the high temperature side the internal energy is $U=\frac{3}{4} T$, from (14), and so approaches the phase transition with a finite slope, giving constant specific heat (15).

This transition has the characteristic features of a 2nd order transition when approached from low temperatures while those of a 1st order transition when approached from the high temperature side. We might call such a transition a 1.5 order phase transition. The two dimensional dimer model has similar asymmetric thermodynamics in the neigbhourhood of its transition. Curiously in the dimer example the background geometry can also be interpreted as undergoing a transition [24].

The free energy per degree of freedom was also derived, in the same approximation as the internal energy above, in [11. On the low temperature side it is 3

$$
\frac{F}{N^{2}}=T\left[\ln \left(\frac{\phi}{T}\right)-\frac{1}{3}\right]-\frac{\phi^{4}}{24} .
$$

Conversely, on the high temperature side, integrating

$$
U(T)=-T^{2} \frac{d}{d T}\left(\frac{F}{T}\right)=\frac{3}{4} T,
$$

leads to

$$
\frac{F}{N^{2}}=C_{1} T-\frac{3}{4} T \ln T
$$

with $C_{1}$ an integration constant. Adjusting $C_{1}$ so that $F\left(T_{c}\right)$ matches on the high and low side gives $C_{1}=\frac{\ln 6}{4}-\frac{7}{12} \approx-0.1354$ and results in the free energy per degree of freedom shown in Fig. 22, There is a jump in the specific entropy, $s=-\frac{1}{3 N^{2}} \frac{d F}{d T}$ as we go through the phase transition, $\Delta s=\frac{1}{9}$.

Of course this classical and semi-classical analysis is not the whole story and indeed the purpose of the present work is to study the characteristics of this phase transition numerically.

\section{$3 \quad$ Finite size critical systems}

Phase transitions where some observables are non-analytic functions of the temperature, $T$ (e.g. they may diverge) are possible only in the thermodynamic

\footnotetext{
${ }^{3}$ One must be careful in specifying the measure when determining the free energy, and the measure for the matrices $X_{a}$ differs from that for the $D_{a}$ by a temperature dependent factor [11. The from of the free energy quoted here is that associated with the $D_{a}$.

${ }^{4}$ Note: There are $3\left(N^{2}-1\right)$ degrees of freedom.
} 


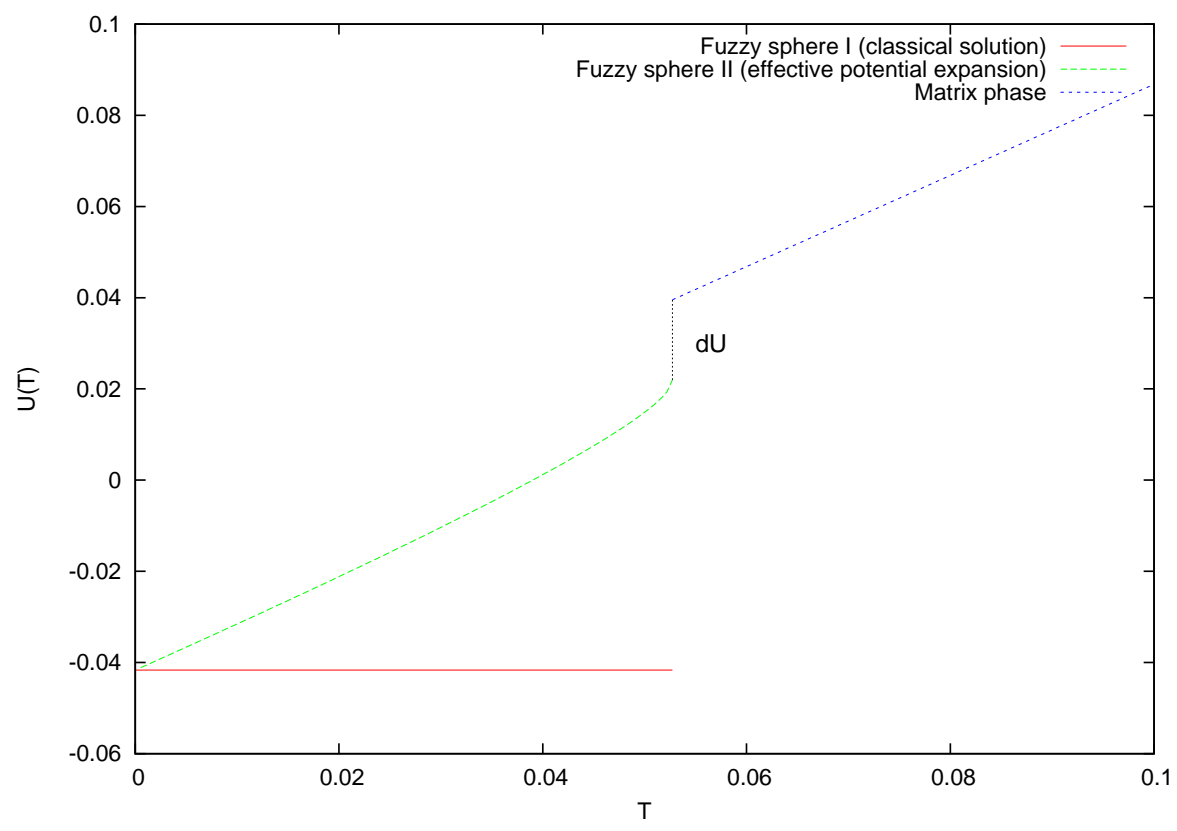

Figure 1: The internal energy per degree of freedom showing critical behaviour on the low temperature side and non-critical behaviour on the high temperature side. The slope near $T_{c}$, gives the specific heat.

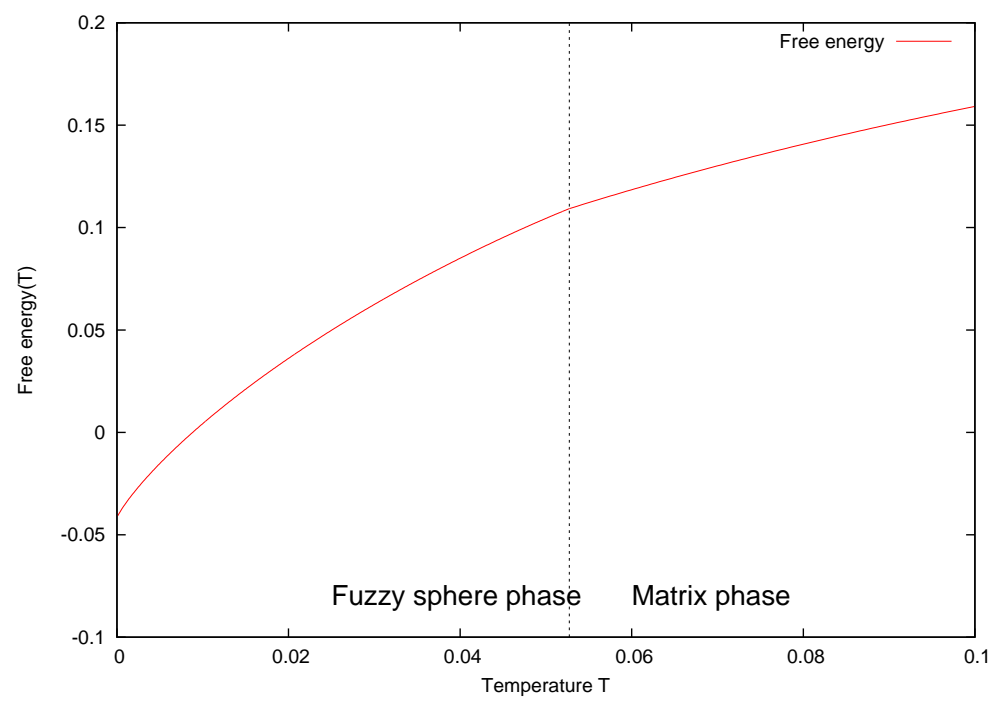

Figure 2: A semi-classical approximation for the free energy per degree of freedom. By construction the free energy is continuous at the critical point, but it is not differentiable, the left derivative is slightly greater than the right derivative, leading to the jump in entropy described in the text. 
limit, which in our case would correspond to taking $N \rightarrow \infty$ at fixed $T$. We can of course only perform numerical studies of systems consisting of a finite number of degrees of freedom, so the systems we simulate will only undergo pseudo phase transitions where the non-analyticities are rounded (e.g. with peaks at pseudo-critical points instead of divergences) 5 Increasing $N$ gets closer to the thermodynamic limit but also increases the computer resources required for the numerical study, truly $N \rightarrow \infty$ systems can only be approximated by finite $N$ systems and the thermodynamic limit must be extrapolated from finite $N$ results. The behaviour of the specific heat as a function of temperature is plotted in Fig. [3 for $N=40$ and $N=100$. The deviation, in the numerical data, of the largest values of $C_{v}$ for different values of $N$ is the due to finite size effects. In Fig. 3 we see that the peak of the specific heat moves with $N$, this is the shift of the pseudo-critical temperature. The temperature at which the different curves begin to deviate from one another corresponds to the rounding temperature and is more difficult to observe in the figure. In Fig. 4 we show a blow-up of the area around the critical point for different values of $N$ where one sees the onset of rounding more clearly. It is probable that the data shown in Figs. 3 and 4 do not achieve the true maximum specific heat since the data do not track the return to low values of the specific heat at high temperatures. However, this is not important for our analysis as we will show that the entire critical regime satisfies scaling with the matrix size.

Before going on to discuss our numerical results in detail we give a brief review of finite size effects on critical systems. See C. Domb and J.L. Lebowitz [15, for further discussion of these issues and for the original literature see also [16].

\subsection{Thermodynamic limit away from the critical point}

Let $F\left(T, N_{d}\right)$ be the free energy for a system with $N_{d}$ degrees of freedom at a temperature $T$. In the thermodynamic limit the free energy per degree of freedom is

$$
f_{\infty}(T)=\lim _{N_{d} \rightarrow \infty} \frac{1}{N_{d}} F\left(T, N_{d}\right) .
$$

In our case $N_{d}=3\left(N^{2}-1\right) \sim N^{2}$. Far away from any critical point we expect this limit to exist and to be independent of the macroscopic geometry.

When the number of degrees of freedom is finite and all degrees of freedom are equivalent, e.g. there are no surfaces and the couplings are isotropic, then there is a characteristic linear system size $L \sim a N_{d}^{\frac{1}{d}}$, where $d$ is the dimension and $a$ is a microscopic scale such as a lattice spacing and the volume of the system is $V=L^{d}$. Since $a$ is fixed, and typically absorbed in the parameters of the system, one can take $a=1$.

\subsection{Finite size effects and the correlation length}

When a finite system approaches a critical regime there is a number of important effects that must be taken into account. First there is a temperature, called the rounding temperature and denoted $T^{*}(L)$, at which observables of the finite

\footnotetext{
${ }^{5}$ For more a comprehensive treatment of critical phenomena the reader is directed to see the reviews in 20, 21, 22, 23.
} 


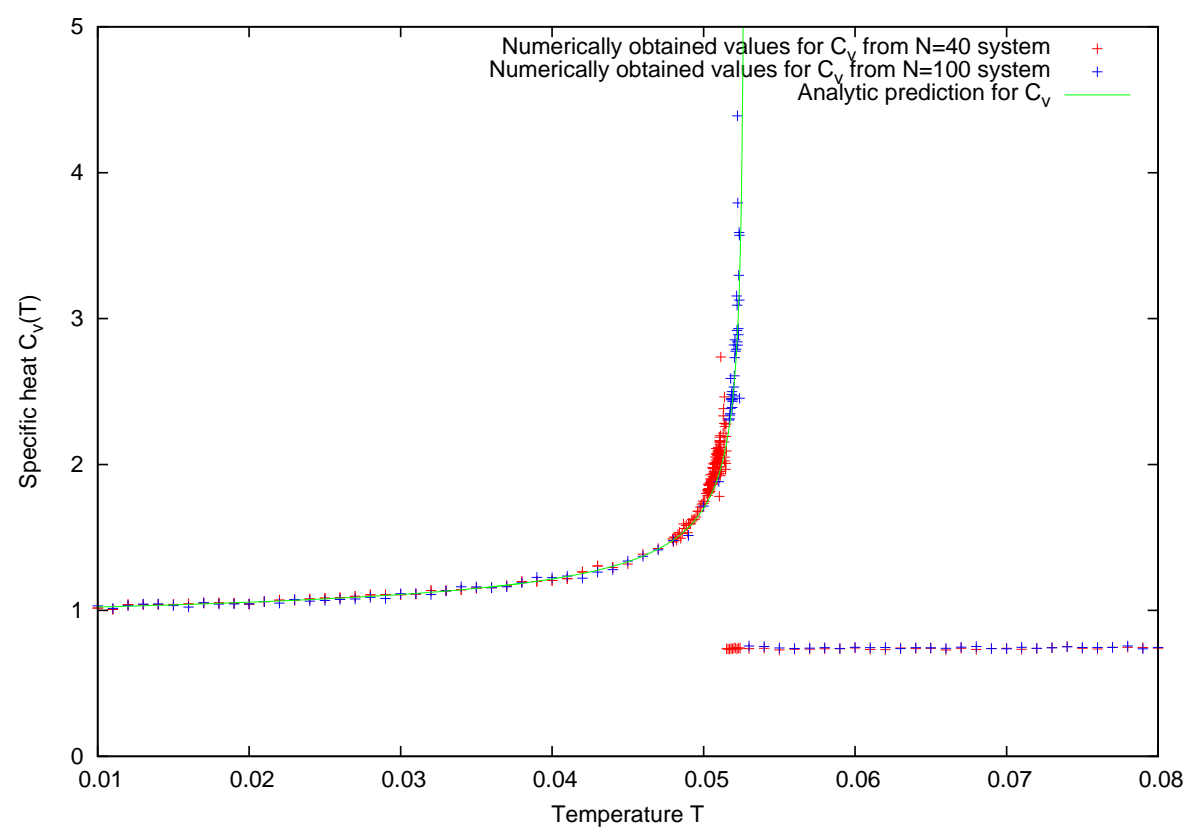

Figure 3: Specific heat for $N=40$ and $N=100$ as a function of temperature (or equivalently, $\widetilde{g}^{-4}$ ), compared to the theoretical prediction (22). The deviation of the numerical data from the theoretical curve, for largest values of $C_{v}$ plotted very near the critical point, is a finite size effect. We can also see that the maximum observable $C_{v}$ for $N=100$ is closer to the transition temperature than that for $N=40$. This is the shift in pseudo critical temperature. 


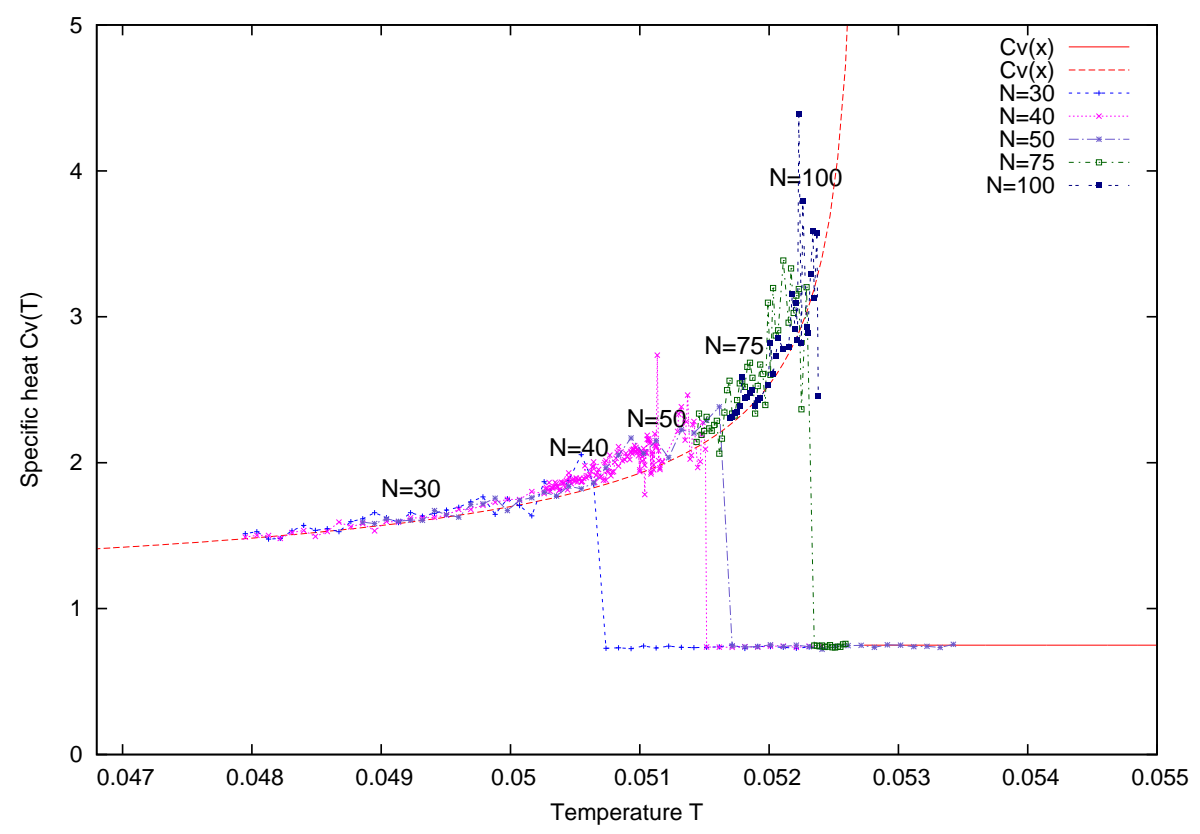

Figure 4: Blow up of specific heat for different system sizes, compared to the theoretical prediction (22).

system start to deviate from those of the infinite system and $T^{*}(L) \rightarrow T_{c}$ as the system size is increased. Finite size scaling assumes that this approach of the rounding temperature to the bulk critical temperature is governed by scaling with

$$
\left|T^{*}(L)-T_{c}\right| / T_{c} \sim A_{\theta} L^{-\theta}
$$

where $\theta$ is the rounding exponent.

Another finite size effect, which is directly visible in Fig. 4, is that thermodynamic quantities which diverge at the critical point merely have maximal values in finite systems with the maximum at some temperature $T_{m}(L) \neq T_{c}$. Such temperatures are called pseudo-critical. Again $T_{m}(L) \rightarrow T_{c}$, as the system size is increased and finite size scaling conjectures that

$$
\left|T_{m}(L)-T_{c}\right| / T_{c} \sim A_{\lambda} L^{-\lambda}
$$

where $\lambda$ is the shift exponent.

The specific heat of many critical systems is one such observable and finite size effects round a divergent specific heat so that it has a maximum,

$$
C_{v m}(L)=C_{v}\left(T_{m}(L)\right)
$$

with $C_{v m}(L) \rightarrow \infty$ as $L \rightarrow \infty$. Finite size scaling implies that the divergence emerges in the limit of infinite $L$ via scaling. Thus

$$
C_{v m}(L) \equiv C_{v}\left(T_{m}(L)\right) \sim A_{\omega} L^{\omega}
$$

The exponents $\theta, \lambda$ and $\omega$, describe the critical behaviour of our finite system as the system size goes to infinity. 
As the critical point of a bulk system is approached the length scale, over which fluctuations in the system are correlated, grows. The correlation length is defined as the rate of the asymptotically exponential decay of the two-point function, with distance, $r$, between the points. For the near-critical system

$$
\Gamma(r, T) \sim \exp (-r / \xi(T)) \text { as } r \rightarrow \infty,
$$

from which the $\xi(T)$ can be computed as

$$
\xi^{-1}(T)=-\lim _{r \rightarrow \infty} \frac{\ln \Gamma(r, T)}{r} .
$$

At a critical point the correlation length diverges and, in most systems, in the immediate vicinity of the critical point $\xi(T)$ can be expressed a 6

$$
\xi(T) \sim f^{ \pm}|t|^{-\nu} \quad \text { where } \quad t=\frac{T-T_{c}}{T_{c}}
$$

is the reduced temperature and the $f^{+}$and $f^{-}$are system dependent amplitudes above and below the critical temperature. They are typically different from one another but their ratio is universal within a given universality class. The exponent $\nu$ therefore dictates how fast the correlation length diverges when $t \rightarrow 0$ in an infinite system.

The correlation length, of a system plays a crucial rôle in the explanation of finite size scaling for systems exhibiting critical phenomena such as the Ising model or gas systems 7 The finite size system correlation length $\xi_{L}$ is constrained by the size of the system and one expects that

$$
\xi_{L}\left(T_{m}(L)\right) \sim L
$$

We can classify the different regimes for the system in terms of the scaled variable $y=\frac{\xi_{L}}{L}$ or $z=\frac{\xi(t)}{L}$. As we approach criticality for finite size system the correlation length takes the form

$$
\xi(T, L)=L y(z)=L f\left(t L^{1 / \nu}\right) .
$$

where both $y$ and $f$ are universal scaling functions 8 .

In this critical regime and when the system is large relative to the correlation length $y \ll 1, y(z) \simeq z$ and $f(x) \sim x^{-\nu}$ so that bulk scaling is recovered. As the critical temperature is approached finite size effects become important and $y(z)$ begins to deviate from $z$. This occurs at the rounding temperature $T^{*}(L)$. For a system of finite extent the correlation length cannot grow arbitrarily large relative to the system size and at the pseudo-critical temperature the specific

\footnotetext{
${ }^{6}$ In fact there are systems where the correlation length diverges faster then any polynomial. A famous example is the Kosterlitz-Thouless Phase Transition where $\xi(T)$ diverges exponentially 25

${ }^{7}$ In the case of matrix models, due to the non-local type of interaction between the entries and the absence of a notion of distance between the elements, we can only speculate on the existence of a unique correlation length.

${ }^{8}$ The non universal scale for the argument of $f$ must be adjusted by convention to get all systems in a universality class to match. Such nonuniversal constants in scaling functions are referred to as "metric factors" and they depend on the microscopic details of the system and are fixed by some system independent convention. Metric factors will not be important for our purposes here, since we have only one system.
} 
heat reaches its maximum value. We take the maximum of the specific heat to define the pseudo-critical temperature and at this temperature we must have that both $y$ and $f$ are $L$ independent constants. Therefore $t_{m} L^{1 / \nu}=$ const, which implies

$$
\left|T_{m}(L)-T_{c}\right| \sim L^{-1 / \nu}
$$

so that the prediction of finite size scaling is therefore that the shift exponent $\lambda=\nu^{-1}$.

If we assume that the only relevant quantity in an expansion around $T_{c}$ is the correlation length, then finite size scaling implies that the free energy, in the vicinity of the critical point, takes the form

$$
\lim _{N \rightarrow \infty, T \rightarrow T_{c}} F\left(T, N_{d}\right)-N_{d} f_{\infty}\left(T_{c}\right)=\mathcal{F}\left(t L^{1 / \nu}\right),
$$

where $\mathcal{F}(x)$ is a universal scaling function that depends only on the universality class of the system. Given this form of the free energy we expect that the exponents $\lambda$ and $\theta$ should be the same, though the amplitudes $A_{\theta}$ and $A_{\lambda}$ may differ.

If we take the large $L$ limit for fixed $t$, from the extensivity of the system we must get

$$
\mathcal{F}\left(t L^{1 / \nu}\right) \sim L^{d} t^{2-\alpha}
$$

which requires $\mathcal{F}(x) \sim|x|^{2-\alpha}$, and we infer that $2-\alpha=\nu d$ which is a well known scaling exponent relation.

We can further take two derivatives of (45) with respect to $t$, and divide by $N_{d}$, to obtain the specific heat in the scaling regime and using $2-\nu d=\alpha$ we obtain

$$
C(T, L)=A_{-} L^{\alpha / \nu} \mathcal{C}(x)
$$

where the amplitude $A_{-}$is extracted to guarantee that $\mathcal{C}$ is a universal scaling function. With $x$ the $L$ independent constant $x_{m}$ at the pseudo-critical temperature we have that $A_{-} \mathcal{C}\left(x_{m}\right)=A_{\omega}$ and the prediction of finite size scaling that $\omega=\frac{\alpha}{\nu}$. Taking $L \rightarrow \infty$ at fixed $t$ takes the scaling function past the rounding temperature and scaling gives that for small $x$ that $\mathcal{C}(x) \sim x^{-\alpha}$ so that we recover $\mathcal{C}(T, L) \sim A_{-} t^{-\alpha}$.

In a fully finite system it is not possible to use the expression (39) for all $L$ and $T$ and it is also difficult to apply to numerical data. Alternative definitions of correlation lengths, such as the second moment correlation length, are useful in this contest, see [15], but the scaling analysis is essentially the same.

\subsection{Scaling in terms of $N$}

Our system has no surface, and all $N_{d}=3\left(N^{2}-1\right)$ degrees of freedom are essentially equivalent. However, in our model the dimensionality, $d$, of the system is only conjectural (we expect $d=2$ on the fuzzy sphere side of the transition). We also do not have access to either a correlation length or a physical notion of size, $L$. However, there is no difficulty in formulating a scaling ansatz in terms of the matrix size, $N$. The essential feature of finite size scaling is then that the system in the critical regime scales with $N$.

Instead of (34), (35), (37) we use:

$$
\left|T^{*}(N)-T_{c}\right| / T_{c} \sim A_{\bar{\theta}} N^{-\bar{\theta}},
$$


for scaling of the rounding temperature with $N$;

$$
\left|T_{m}(N)-T_{c}\right| / T_{c} \sim A_{\bar{\lambda}} N^{-\bar{\lambda}}
$$

for scaling of the shift with $N$ and

$$
C_{v m}(N):=C_{v}\left(T_{m}(N)\right) \sim A_{\bar{\omega}} N^{\bar{\omega}} .
$$

for scaling of the peak in the specific heat with $N$.

One can repeat the analysis of the previous section but now using $N$ rather than $L$. Scaling suggests that (50) for the free energy of the system should be replaced by

$$
\lim _{N \rightarrow \infty, T \rightarrow T_{c}} F(T, N)-N^{2} f_{\infty}\left(T_{c}\right)=\mathcal{F}\left(t N^{\bar{\lambda}}\right)
$$

and we take the scaling variable9 to be $x=t N^{\bar{\lambda}}$. Taking the infinite $N$ limit for fixed $t$ gives $\mathcal{F}(x) \sim|x|^{2-\alpha}$ and $(2-\alpha) \bar{\lambda}=2$. If we input the theoretical prediction for $\alpha=\frac{1}{2}$ we have the further prediction that

$$
\bar{\lambda}=\frac{4}{3} .
$$

Furthermore, once the specific heat has risen sufficiently above the background values that arise far from $T_{c}$, we expect that it has the form

$$
C(T, N)=N^{\bar{\omega}} A_{\bar{\omega}} \mathcal{C}(x)
$$

where $\mathcal{C}(x)$ is a universal scaling function. The scaling function $\mathcal{C}(x)$ must have the behaviour $\mathcal{C}(x) \sim x^{-\alpha}$ for large $x$. Also for $T=T_{m}(N)$ we require that $x=x_{m}$ be independent of $N$, since $C_{v m}$ is given by (49) and hence

$$
\bar{\omega}=\bar{\lambda} \alpha=\frac{2}{3} .
$$

The value $\mathcal{C}(0)$ is a universal number for our system, but it is difficult to evaluate with any precision due to the difficulties of accessing this region of parameter space for large matrix sizes.

The relation (51) and (53) are derived without reference to a correlation length or any other notion of distance and cannot be used to measure the dimensionality or a characteristic size of the system. The scaling above is important to us because it contains only exponents that are directly accessible to our numeric measurements and can be used to test finite-size scaling in the context of the current matrix model.

By definition the rounding temperature, $T^{*}(N)$, is that temperature where deviations from the asymptotic scaling form begin. As $N$ is increased $T^{*}(N)$ moves closer to the transition temperature. There is, however, no unambiguous connection between $T^{*}(N)$ and $N$. The scaling function $\mathcal{C}(x)$ should be analytic, aside from its asymptotic form at large argument, so one can replace $T$ in (52) either with $T^{*}(N)$ from (47) or $T_{m}(N)$ from (48) and both should give a specific heat that diverges with $N$ as $N^{\bar{\omega}}$ but with different amplitudes. Therefore we expect $\theta=\lambda$ and $\bar{\theta}=\bar{\lambda}$.

\footnotetext{
${ }^{9}$ We have set the metric factor here to 1 for convenience here.
} 
If we take the standard relation that $L=a N_{d}^{1 / d}$, which for us gives $L=$ $N^{2 / d}$, assuming the microscopic scale $a=1$ then

$$
\bar{\theta}=\theta \frac{2}{d}, \quad \bar{\lambda}=\lambda \frac{2}{d}, \quad \bar{\omega}=\omega \frac{2}{d} .
$$

If we further assume the existence of a single correlation length, $\xi(T)$, dominating the critical region then we have

$$
\bar{\lambda}=\bar{\theta}=\frac{2}{d \nu} .
$$

We cannot draw a confident conclusion about $\nu$ and $d$ separately as our analysis only gives the product $d \nu$ via measurements of $\bar{\lambda}$ and $\bar{\omega}$. However, if we assume that $d=2$, which seems reasonable based on the fuzzy sphere as background geometry, we have the prediction for the correlation exponent

$$
\nu=\frac{3}{4} \text {. }
$$

We now turn to the numerical measurements.

\section{Near-critical simulation difficulties}

In this section we discuss some of the challenges posed in a numerical analysis of the properties of the system very close to the phase transition and describe how they are tackled.

\subsection{Critical Slowing Down}

Critical slowdown is a phenomenon that is typical in numerical simulations of critical systems. It is described by the theory of dynamic critical phenomena (see e.g. [23, 27] for detailed treatments of the subject). When $T \rightarrow T_{c}$ the time needed for a non-equilibrium system to reach equilibrium grows as does the auto correlation time in a Monte Carlo simulation of the system. More detail on the impact of critical slowdown on our numerics is discussed in the Appendix and here we merely observe that, for the systems studied in this work, the critical slowing down of our simulations has significant impact on systems with $N \geq 100$ and has prevented us from simulating matrices with $N>11010$

\subsection{Excited states}

Another property to be taken into consideration is the presence of the excited fuzzy sphere configurations given by (19). As mentioned earlier, those configurations possess energies which are intermediate between the commuting matrix phase and the fuzzy sphere phase. This means that, in the region where the two phases coexist, we would expect to see jumps between the ground state and these excited states and between these excited states and the commuting matrix phase rather than direct transitions between the commuting matrix phase and the fuzzy sphere ground state. This expectation is supported by our simulations.

\footnotetext{
${ }^{10}$ One can see in Fig. 9 that the relative error in the measurement of the near-critical specific heat grows with the system size.
} 


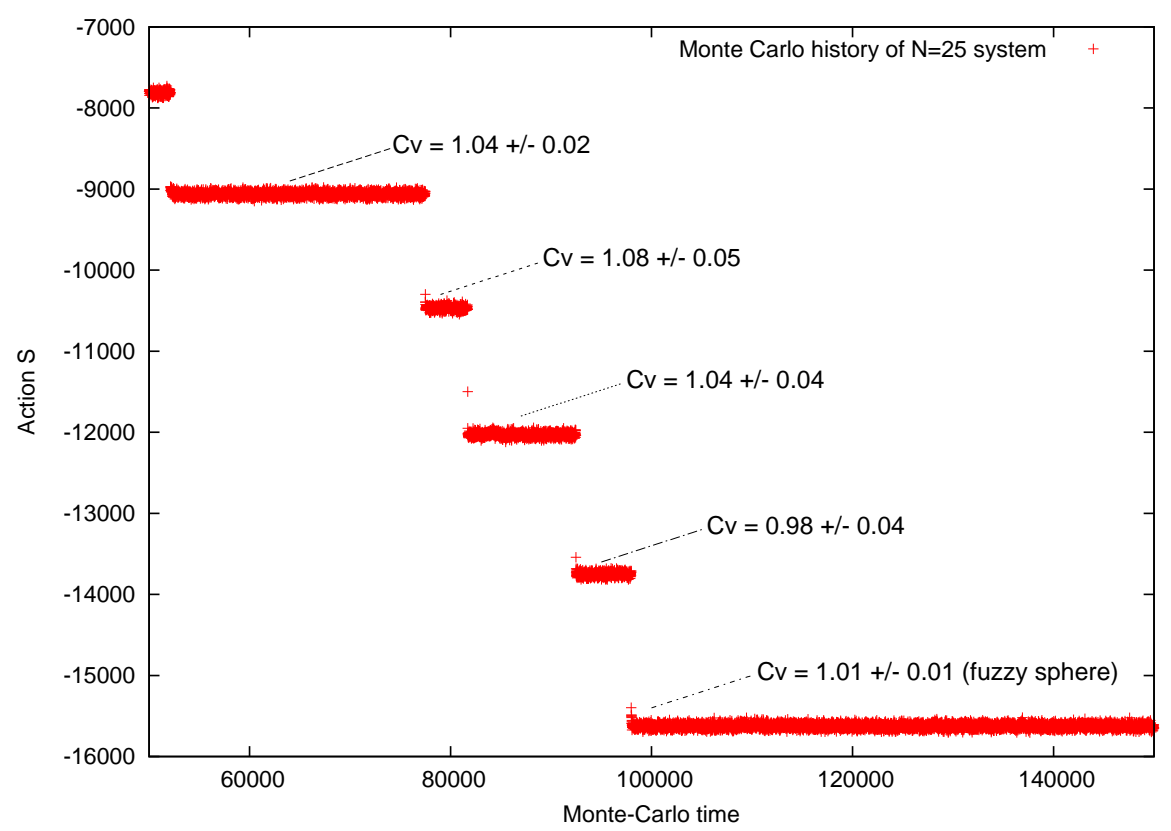

Figure 5: Monte Carlo history of an $N=25$ system visiting different excited states, together the corresponding $C_{v}$ in their specific domains (for $\widetilde{g}=5$, i.e. $T=0.0016$ and $\left.\frac{T}{T_{c}}=0.03\right)$.

Far from the transition we are able to measure the values of $C_{v}$ for restricted ensembles trapped in different excited states and for the fuzzy sphere ground state. We find that sufficiently far from the transition all such specific heats are of order 1 i.e. $C_{v} \sim 1$ as illustrated on Fig. 5 . As the transition is approached $C_{v}$ grows and close to the transition distinct states are no longer observable see Fig. 6.

\subsection{Energy separation between different phases}

Ideally in the vicinity of the phase transition the system will jump between the two phases and if we can get enough Monte Carlo steps, we will have enough statistics to properly extract the relevant quantities. A Monte Carlo history where this occurs is depicted in Fig. 6. We can see that the system spends roughly the same amount of MC time in both phases, an indication that the system is close to the transition point. However for large $N$ the jump between the two phases becomes rare events, and indeed our numerical studies indicate that this is already the case for $N \simeq 12$. This, in combination with the asymmetry of the phase transition, makes it very hard to simulate the system efficiently in the regime where the two phases coexist.

One tactic to handle this problem is to perform a cold start on the Monte Carlo runs, so that the phase transition is always approached from the low temperature side. This biases the system toward the fuzzy sphere phase, but has the advantage of giving reproducible results. An example is shown in Fig. 7 , with $N=50$ at $T=0.0514<T_{c}$. The system is below the critical temperature 


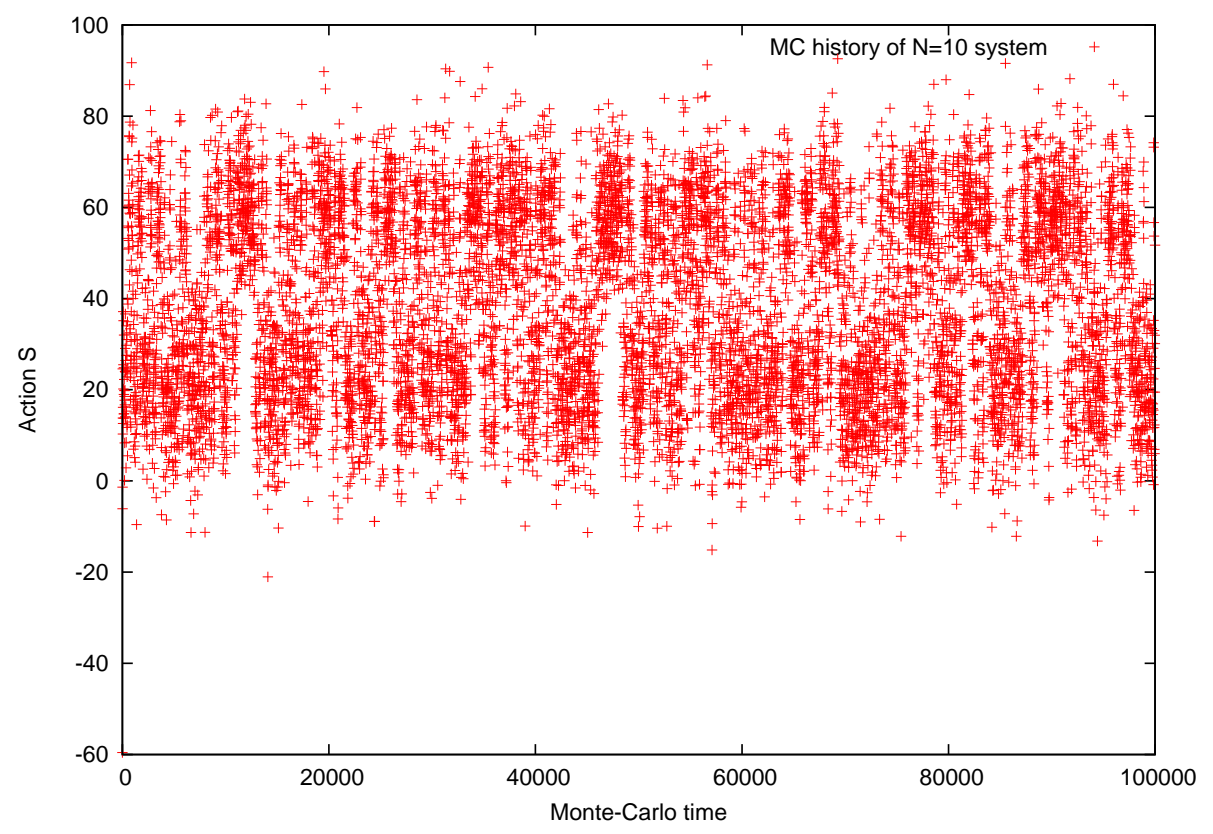

Figure 6: Monte Carlo history of an $N=10$ system at $T=0.0468$, a regime where the two phases coexist. For these values of $N$ and $T$ equation (14) gives $S_{m} \approx 75$ while (27)-(29) give $S_{f} \approx 20$ and the action is seen to jump randomly in the vicinity of, and between, these two values.

but, while the value of the action is compatible with a fuzzy sphere configuration for quite some Monte Carlo time, it suddenly jumps to a commuting matrix configuration. Once in the matrix phase configuration, it remains there as fluctuations are too small to get it back.

\subsection{Comments on the algorithm}

When starting the system in a zero field configuration, and using a simple Monte Carlo simulation, the system tends to get stuck in the zero action local minimum. The typical configuration that such simulations produce is one where two of the matrices have zero eigenvalues while those of the third matrix acquires non-zero eigenvalues which are distinct from one another. The matrix that is started first in the simulation is the one whose eigenvalue become non-zero and non-degenerate. A hybrid Monte Carlo simulation is necessary to overcome this difficulty and for our study we use the hybrid Monte Carlo algorithm of [26].

It is also tempting to perform simulations for the system by first diagonalising one of the matrices. This results in a Vandermonde Jacobian from the change of variables whose logarithm is included in the effective action. This algorithm is quite efficient for some simulations but simulations with a hot start find it much more difficult to relax to a fuzzy sphere phase even for very low temperatures and relatively large matrices. This is because separation of the eigenvalues from one another must filter from the outer eigenvalues inwards which tends to be very slow. To avoid any such difficulties we have chosen to 


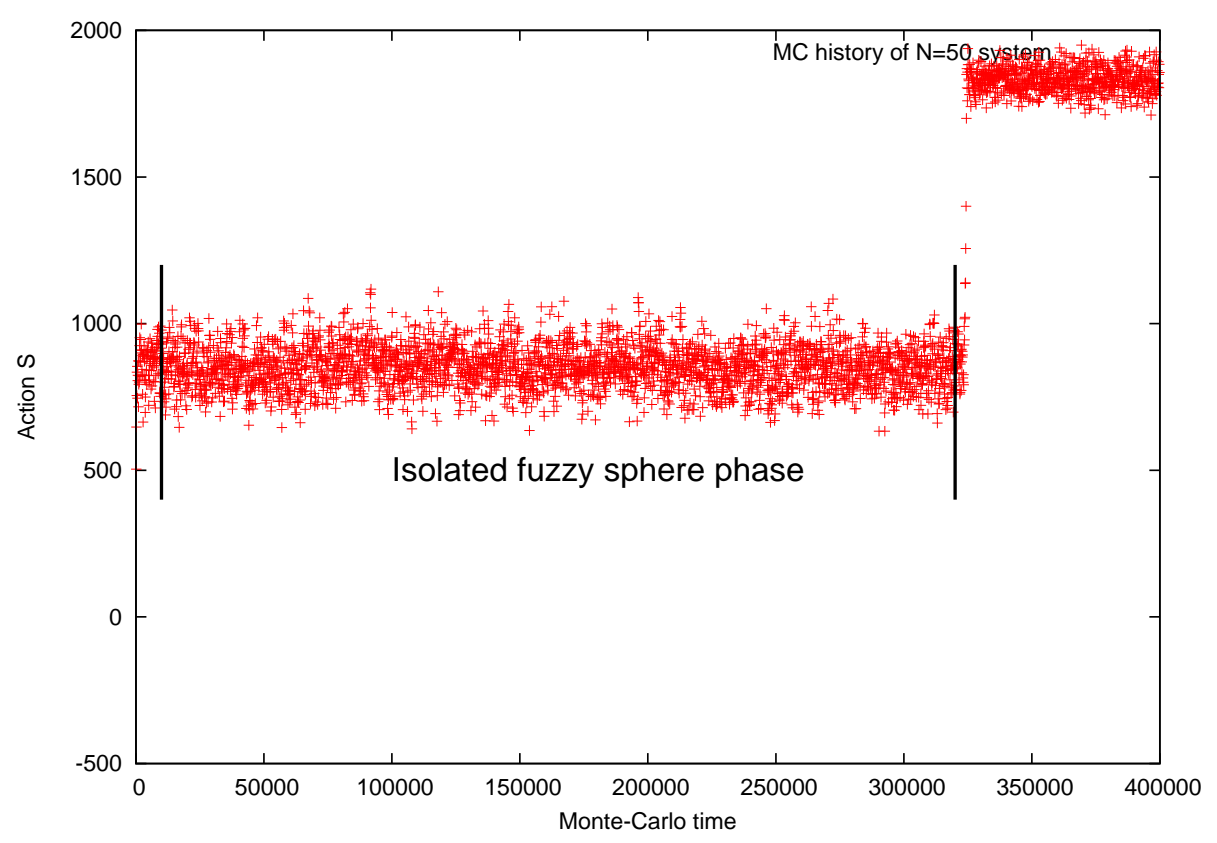

Figure 7: Monte Carlo history of an $N=50$ system at $T=0.0514$ crossing between the fuzzy sphere phase and the commuting matrix phase as an illustration of the restricted ensembles approach. For these values of $N$ and $T$ equation (14) gives $S_{m} \approx 1875$ while (27)-(29) give $S_{F} \approx 850$. The vertical bars show the fuzzy sphere domain, if we restrict our measurements only to this region we can extract observables in the fuzzy sphere phase. These are what we call restricted ensemble measurements. 
use the direct approach and our simulations are performed with a hybrid Monte Carlo algorithm in which all matrices are treated on an equal footing.

\section{Numerical measurement of critical exponents and finite size scaling}

A direct analysis of the specific heat data, in the immediate vicinity of the critical point, would involve a four parameter fit

$$
C_{v}(T)=C_{0-}+A_{-}\left(T_{c}-T\right)^{-\alpha}
$$

where the data are fit to obtain $C_{0-}, A_{-}, T_{c}$ and $\alpha$. This, however, involves large errors as there are so many parameters and the specific heat data for $T$ very close to $T_{c}$ involves finite size effects which mean that the finite size scaling function $\mathcal{C}(x)$ enters the picture. The first step is therefore a the determination of the critical temperature.

\subsection{Estimating $T_{c}$ and $\bar{\lambda}$ and $\bar{\omega}$}

A precise determination of the critical temperature is necessary for the evaluation of the specific heat exponent $\alpha$ and the shift exponent $\bar{\lambda}$. For a given finite $N$ our best estimate of the critical temperature is the pseudo-critical temperature $T_{m}(N)$. We therefore first analyse $T_{m}(N)$ and endeavour to extract $T_{c}$ from the limit of $T_{m}(N)$ for $N$ going to infinity by fitting it as a function of $N$ to the shift scaling form (48)

$$
T_{m}(N)=T_{c}\left(1-A_{\bar{\lambda}} N^{-\bar{\lambda}}\right) .
$$

In Fig. 8 we present simulation our data for $T_{m}(N)$.

Visual inspection of the data suggests a linear fit, i.e. $\bar{\lambda}=1$. Linear regression on the data in Fig. 8 gives $T_{c}=0.0532 \pm 0.0001$ and $A_{\bar{\lambda}}=1.5 \pm 0.1$ but assumes $\bar{\lambda}=1.0$. However, the scaling ansatz suggests that we should look for a three parameter fit. When we perform such a three parameter fit we get $T_{c}=0.0531 \pm 0.0003, A_{\bar{\lambda}}=1.8 \pm 1.5$ and $\bar{\lambda}=1.1 \pm 0.3$ and the resultant critical temperature from both fits is largely unchanged.

For the three parameter fit the error in the amplitude $A_{\bar{\lambda}}$ is rather large so it is desirable to fix some of the parameters. Since our principal goal is to check scaling we need to measure $\bar{\lambda}$ rather than assume it. The measurements of $T_{c}$ from both the three parameter fit and the linear one broadly agree with the theoretical prediction $T_{c}=\left(\frac{3}{8}\right)^{3} \simeq 0.0527344$, suggesting that we constrain the fit so that $T_{c}$ is fixed to be this number. With $T_{c}$ so constrained we then find a two parameter gnuplot fit gives $A_{\bar{\lambda}}=5.2 \pm 1.6$ and $\bar{\lambda}=1.41 \pm 0.08$.

Finally since we have a prediction for the exponent $\bar{\lambda}$, see (51), fixing both $T_{c}$ and $\bar{\lambda}=\frac{4}{3}=1.333$ gives $A_{\bar{\lambda}}=3.9 \pm 0.1$ suggesting perhaps that $A_{\bar{\lambda}}=4$. We conclude that the data are quite consistent with the theoretical estimate though a linear fit is also consistent with our measurements.

We can similarly analyse the maximum of the specific heat $C_{v m}(N)$. A linear fit assumes $\bar{\omega}=1$ and is best interpreted as an estimation of $C_{0-}$, our data from such a linear fit gives $C_{v m}=0.8 \pm 0.2+(0.035 \pm 0.002) N$ which gives 


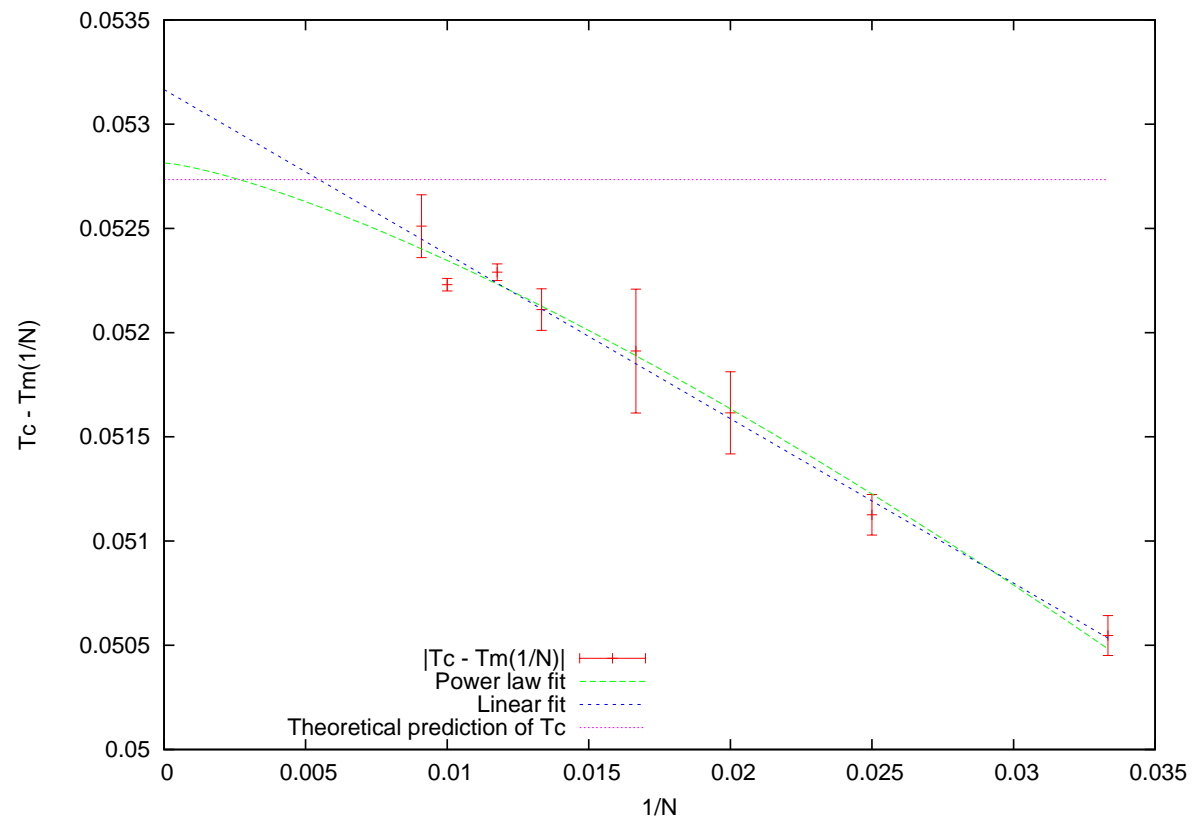

Figure 8: Extrapolation of $T_{m}(N)$ for $\frac{1}{N} \rightarrow 0$. The value at 0 corresponds to $T_{m}(\infty)$ which is $T_{c}$. The theoretical prediction is given by the blue dashed line. The green line is a three parameter fit to equation (48) with $T_{c}=0.0531 \pm 0.0003$, $A_{\bar{\lambda}}=1.8 \pm 1.5, \bar{\lambda}=1.1 \pm 0.3$ 


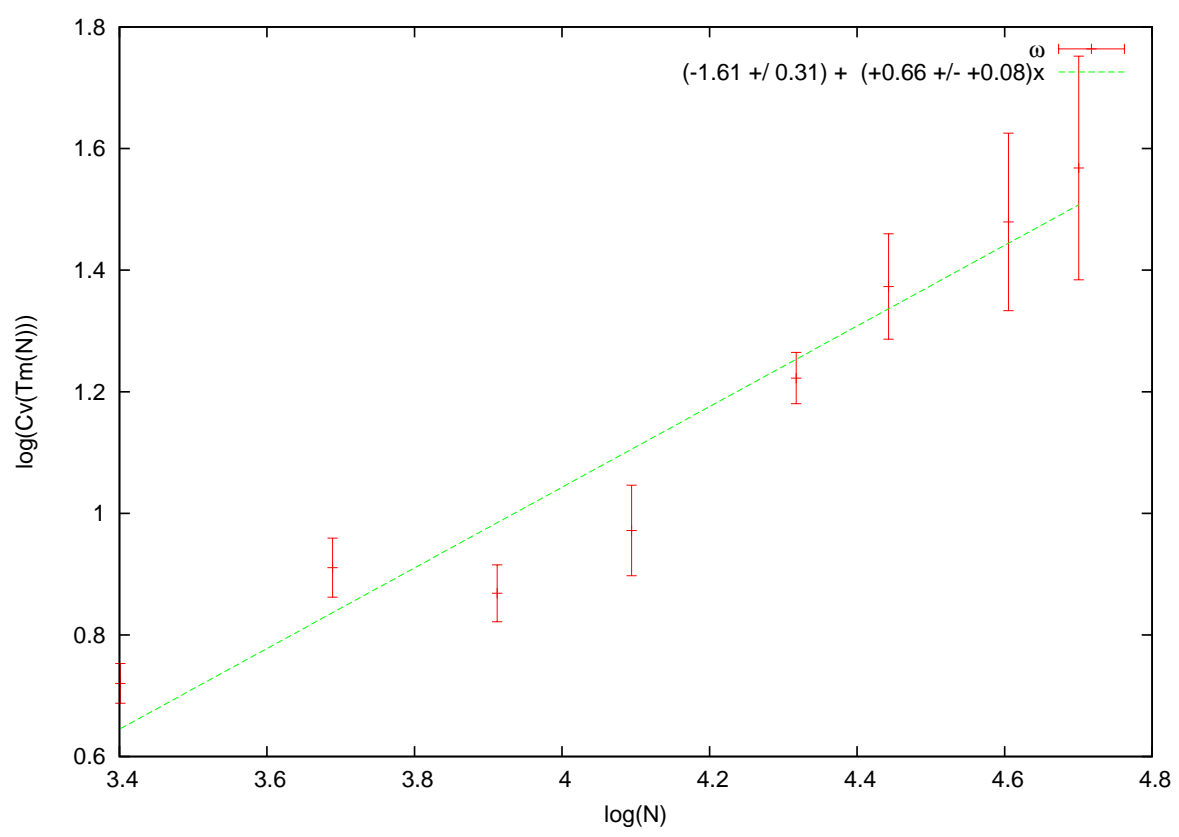

Figure 9: A plot of $C_{v}\left(T_{m}(N)\right)$ as a function of $N$, on a double-logarithmic scale, and least squares fit to data. The slope of the linear fit corresponds to $\bar{\omega}$ in (49) for $C_{v}$ and gives $\bar{\omega}=0.66 \pm 0.08$.

a value of $C_{0-}$ consistent with the theoretical prediction of $\frac{29}{36} \simeq 0.8055$. A three parameter fit gives $C_{v m}=(1.7 \pm 0.3)+(0.001 \pm .002) N^{1.7 \pm 0.4}$, but the amplitude is very small and has large errors. Our scaling ansatz suggests that we should look for a fit $C_{v m}(N)=A_{\bar{\omega}} N^{\bar{\omega}}$ which is best extracted from a log-log plot. Such a log-log plot is shown in Fig. 9] and gives $A_{\bar{\omega}}=0.21 \pm 0.06$ and $\bar{\omega}=0.66 \pm 0.08$ which is surprisingly close to the theoretical prediction $\bar{\omega}=\frac{2}{3}$.

Summarising our data: When the critical temperature is assumed to be the theoretical value we find with two parameter fits the amplitudes and exponents in (48) and (49) are given by

$$
\begin{gathered}
\frac{T_{c}-T_{m}(N)}{T_{c}} \sim(5.2 \pm 1.6) N^{-1.41 \pm 0.08} \\
C_{v}\left(T_{m}(N)\right) \sim(0.21 \pm 0.06) N^{0.66 \pm 0.08} .
\end{gathered}
$$

where we have chosen to prefer the direct fit for $T_{m}(N)$ and the logarithmic fit for $C_{v m}(N)$. The basic data used are shown in Fig. 8 and 9

The data yield the exponents $\bar{\lambda}=1.41 \pm 0.08$ and $\bar{\omega}=0.66 \pm 0.08$ and the scaling relation $\alpha=\bar{\omega} / \bar{\lambda}$, see eqn. (53), predict $\alpha=0.47 \pm 0.06$ which within errors is consistent with $\alpha=0.5$.

\subsection{Direct measurement of $\alpha$ from collapsed data}

As argued in the previous section the numerical estimate for $C_{0-}$ in (26) when extracted from a linear fit to the specific heat maximum, is in good agreement 
with the theoretical prediction (25) $C_{0-}=\frac{29}{36}$. It is then reasonable to take this as the input value of $C_{0-}$ and endeavour to extract the amplitude and exponent $\alpha$ from the data.

However, data for a given fixed $N$ are not very satisfactory for the estimation of $A_{-}$and $\alpha$ since any such data have important finite size corrections for finite $N$, i.e. the presence of values of the specific heat taken at temperatures $T<T^{*}(N)$ when extracting the exponent from single matrix size data. As we pointed out earlier, the point $T=T^{*}(N)$ is hard to detect. Since the rounding temperature depends on $N$, we can do better by combining different values of $N$. Deviations due to the rounding effects are significantly diminished by averaging over the specific heat for different $N$.

Scaling suggests that, for a fixed value of the temperature lower than $T^{*}(N)$, the values of the specific heat should be consistent for different $N$, provided $N$ is large enough, and our measurements verify this expectation. So we treat such values as independent measurements of $C_{v}(T)$ and then take the weighted average over such values. If we have a number of independent measurements of $C_{v}$, labeled by a discrete index $i$ for different values of matrix size $N_{i}$, then the weighted average, $C_{v}(T)$, is defined to be

$$
C_{v}(T)=\frac{\sum_{i} \frac{1}{\sigma_{N_{i}}^{2}} C_{v}\left(T, N_{i}\right)}{\sum_{i} \frac{1}{\sigma_{N_{i}}^{2}}}
$$

where $\sigma_{i}$ is the uncertainty in measurement $i$. Fig. 10 plots a weighted average $C_{v}$ using data corresponding to values of $N$ ranging from 30 to 110 .

Fig. 10 shows combined data for different $N$. When we approximate the near-critical behavior of the specific as $C_{v} \sim C_{0-}+A_{-}\left|T_{c}-T\right|^{-\alpha}$ and use a three parameter fit we find $C_{0-}=0.76 \pm 0.09, A_{-}=0.051 \pm 0.017$ and $\alpha=-0.50 \pm 0.04$. All three parameters are in very good agreement with (25). One might expect $C_{0-}$ to be the least sensitive of the parameters in the approach to the singularity, and it is tempting to set $C_{0-}$ to the theoretical background value and refine the estimate of $A_{-}$and $\alpha$. When this is done we find $A_{-}=$ $0.043 \pm 0.0014$ and $\alpha=0.5197 \pm 0.0054$. If we also set $\alpha=\frac{1}{2}$ for a one parameter fit we get $A_{-}=0.0487 \pm 0.0002$ which suggests that the true value is indeed $A_{-}=\frac{3}{64}=0.046875$. It should be noted that the form (25) is only asymptotic and has additional corrections. Also our data still has finite size effects included. As a final estimate we set $C_{0-}=\frac{29}{36}$ and perform a two parameter direct fit to the data we get $A_{0-}=0.047 \pm 0.001$ and $\alpha=0.51 \pm 0.01$. In summary we believe that our data gives reasonable evidence that (25) captures the true large $N$ behaviour of the system.

On the high temperature side of the transition we find no divergence of the specific heat, and our numerical measurements show good agreement with the value $C_{v}=\frac{3}{4}=$ const. To conform to the standard scenario of critical phenomena there are two alternatives: either $A_{+}=0$ or $\alpha=0$ for $T>T_{c}$. If we assume the critical exponent is equal on the two sides of the transition we are led to the conclusion that $A_{+}=0$. Then we have the universal ratio $U_{0}=A_{+} / A_{-}=$ 0 , the amplitudes $A_{+}$and $A_{-}$are system and/or interaction dependent, but $U_{0}$ is universal. This provides important information for determining the universality class of the system. 


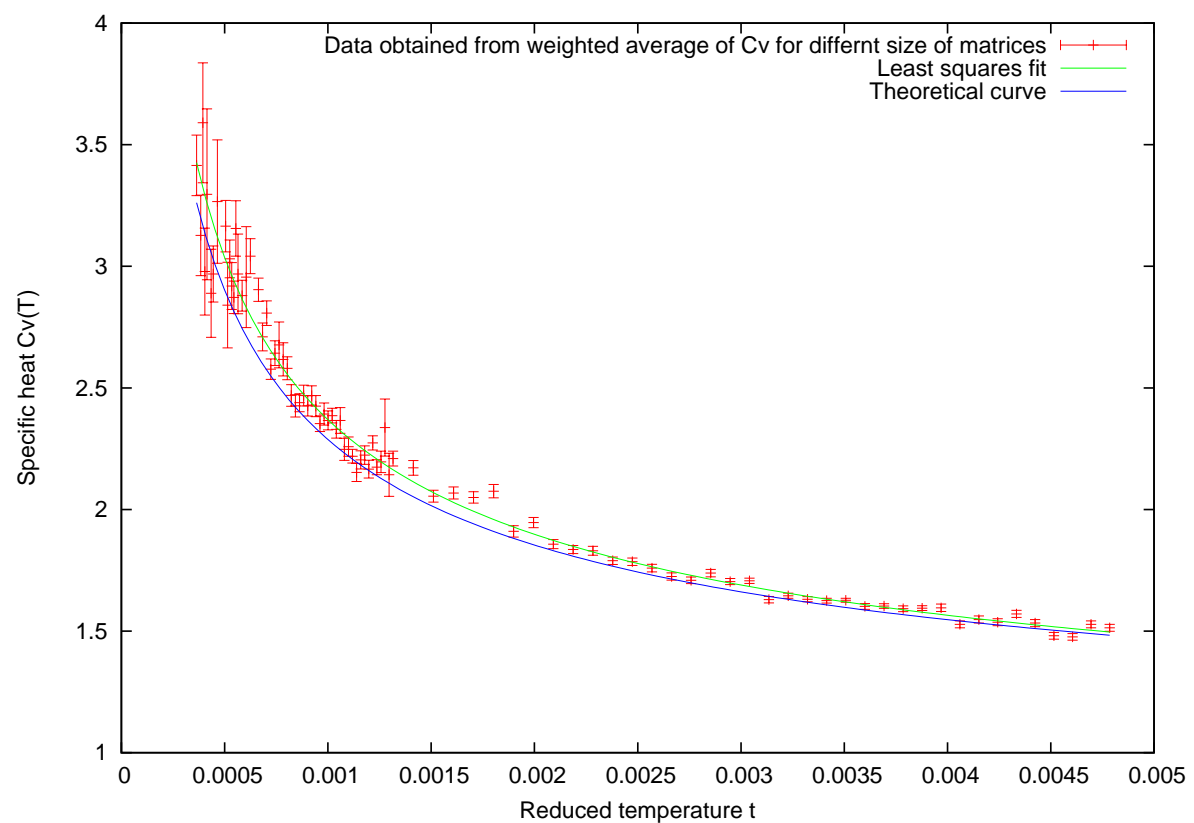

Figure 10: The figure shows the weighted averaged $C_{v}$ obtained from different values of $N$ ranging from 30 to 110 . A three parameter fit to the data with $C_{v}=C_{0-}+A_{-}\left(T_{c}-T\right)^{\alpha}$ gives $C_{0-}=0.76 \pm 0.09, A_{-}=0.051 \pm 0.017$ and $\alpha=-0.50 \pm 0.04$. 


\section{Conclusions and outlook}

One of the key features of our simulations is that the matrix phase configurations tend to be extremely stable. Fluctuations around these are small, they have a restricted ensemble specific heat of $C_{v}=\frac{3}{4}$ in comparison with the fuzzy sphere background whose minimum specific heat is $C_{v}=1$. This means that the decay of matrix phase configurations becomes very unlikely even very close to the transition. The small fluctuation analysis of section 2.1 suggests that the principal mode of decay of such configurations is via the negative eigenvalue identified in section 2.1 and due to the Myers term. The largest eigenvalue separation of matrix phase configurations is $\Delta_{i j}^{a}=2 R$ and such configurations have eigenvalues described by the parabolic distribution (16) so one would expect the matrix phase to become unstable if on average the eigenvalues of one of the matrices are too close together. Taking the expectation value of (11), assuming a parabolic distribution of the eigenvalues, one obtains that the matrix phase becomes unstable for $g^{2}>g_{m}^{2} \simeq \frac{1}{3} \frac{\left\langle\Delta_{i j}^{2}\right\rangle}{4}=\frac{R^{2}}{10}$, where the factor of $1 / 3$ comes from averaging over the number of matrices. For $\tilde{g}$ independent of $N$ this gives $\tilde{g}_{m} \simeq \frac{R \sqrt{N}}{\sqrt{10}}$. So the matrix phase becomes more and more stable as $N$ increases. Noting that numerically $R=2.0$ we find that $\tilde{g}_{m} \simeq 0.632 \sqrt{N}$ so that for $N<11$ we have $\tilde{g}_{m}(N)<\left(\frac{8}{3}\right)^{\frac{3}{4}}$ while for $N \geq 11$ it is larger. For large $N$ and fixed $\tilde{g}$ we therefore expect simulations to get trapped in the matrix phase if the simulation ever falls into such configurations and we further expect that for matrix sizes much larger that $N=11$ it will be virtually impossible to escape from the matrix phase. This is precisely what we observe in simulations. We find that with effort one can escape from the matrix phase for $N \leq 14$ but our simulations have great difficulty escaping for $N=15$ and do not escape for larger $N$. In fact older numerical simulations [6] give the instability of the matrix phase as $g_{m}=0.66$ which is consistent with our observations.

Simulations on small matrix sizes then have quite a different character to those for large matrix sizes. They exhibit fluctuations that make transitions between the two types of typical configuration-the fuzzy sphere and the matrix phase. For larger $N$ such fluctuations are absent and one is forced to take data in a restricted ensemble where the fluctuations are either around the fuzzy sphere or around the matrix phase.

In this work we have not endeavoured to study small enough systems where fluctuations between the matrix phase and the fuzzy sphere are possible. We have rather concentrated on larger matrix sizes as our goal is to check finite size scaling of fluctuations with $N$. Our simulations therefore probe the scaling properties of fluctuations in the fuzzy sphere phase. The fluctuations grow with $N$ and diverge in the large $N$ limit at a critical temperature with the large $N$ fuzzy sphere becoming unstable at $\tilde{g} \simeq\left(\frac{8}{3}\right)^{3 / 4}$ corresponding to a critical temperature $T_{c} \simeq\left(\frac{3}{8}\right)^{3}$. These fluctuations, since they do not probe the matrix phase configurations, are what we call restricted ensemble fluctuations.

It may be that the true thermodynamically stable ground state of the system is in fact the matrix phase, and simulations on small matrix sizes tend to suggest this, however, in the large $N$ limit and for $T<\left(\frac{3}{8}\right)^{3}$ fluctuations never escape from the vicinity of the fuzzy sphere. If the fuzzy sphere phase is only a local minimum and not a global minimum in the vicinity of this transition, then it 
would be clear why the transition appears to be one-sided - the interpretation would be that the system is trapped in a local minimum which is stable due to the large $N$ limit and the observed transition would be due to the configurations eventually escaping over the barrier once the temperature is high enough. The transition would then be very similar to the two dimensional quantum gravity transition discussed in the matrix model literature [28] which also has a one sided transition. The quantum gravity system is exactly solvable and in the case of four valent planar graphs where the potential is

$$
V(\Phi)=N \operatorname{Tr}\left(\frac{1}{2} \Phi^{2}-\frac{g}{4} \Phi^{4}\right)
$$

the eigenvalue distribution is given by

$$
\rho(x)=\frac{1}{2 \pi}\left(1-g \frac{a^{2}}{2}-g x^{2}\right) \sqrt{a^{2}-x^{2}} \quad \text { with } \quad a^{2}=\frac{2}{3 g}(1-\sqrt{1-12 g})
$$

and the specific heat by

$$
C_{v}=\frac{1+54 g^{2}-(1+6 g) \sqrt{1-12 g}}{216 g^{2}} .
$$

By rescaling $\Phi \rightarrow \varphi / \sqrt{g}$ one can rewrite $V(\Phi)=\frac{N}{g} \operatorname{Tr}\left(\frac{1}{2} \varphi^{2}-\frac{1}{4} \varphi^{4}\right)$ and we can be interpreted the coupling as temperature, $g=T$. The system has a critical temperature $T_{c}=g_{c}=\frac{1}{12}$ and a non-analytic specific heat $C_{v}=\frac{11}{12}+$ $\sqrt{12} \sqrt{T_{c}-T}+\cdots$ corresponding to specific heat exponent $\alpha=-\frac{1}{2}$. As in our case, a restricted ensemble, where the system is confined to the well near the origin, will capture this behaviour. The critical point is when the eigenvalues spread enough to spill over the barrier.

As far as our simulations are concerned we start them in, or near, the fuzzy sphere ground state and, for $T \leq\left(\frac{3}{8}\right)^{3}$ and sufficiently large $N$, they almost never escape from this. Our principal observations are restricted to this regime and in this context we have demonstrated that, despite the non-locality of matrix actions and the absence of a characteristic size, finite-size scaling, with matrix size $N$, is still valid.

We have measured the critical temperature and the specific heat critical exponent, $\alpha$, along with the finite-size scaling exponents $\bar{\omega}$ and $\bar{\lambda}$ near the phase transition. Our numerical analysis is compatible with the hypothesis that finitesize scaling is valid, see 3.3 for details.

The values obtained for these exponents are new and we know of no other system with these exponents which suggest that the model under study belongs to a new universality class. We expect that the critical exponents are universal within the class of models with fuzzy sphere geometries evaporating.

Since we have not measured a correlation length and associated critical exponent we cannot establish that our shift exponent $\bar{\lambda}$ is related to the the correlation exponent. The measurement of a correlation length exponent is a non-trivial exercise in this context.

In order to verify the relations (55), i.e. $\bar{\lambda}=\frac{2}{d \nu}$, one needs to measure the exponent $\nu$ for some appropriately defined effective correlation length $\xi(T)$. It seems plausible that a correlation length could be extracted by studying the fall-off of two point correlators, but this is left to future work.

Acknowledgements: We wish to thank Thomas Kaltenbrunner for many helpful discussions. 


\section{A Critical Slowing Down}

In numerical calculations critical slowdown manifests itself as a growth in the number of consecutive measurements of an observable $P$ which are correlated as the transition is approached, i.e. the autocorrelation time $\tau_{P}$ grows as the transition is approached.

Consider a simulation consisting of $N_{m c}$ Monte Carlo steps producing a set of measurements $\left\{P_{1}, \ldots P_{N_{m c}}\right\}$. If $\tau_{P} \leq 1$ an expectation value $\langle P\rangle$ can be computed and assigned an uncertainty $\sigma \sim 1 / \sqrt{N_{m c}}$. When $\tau_{P}>1$ this error estimation is too optimistic, because the measurements are not fully independent, and a better estimation is given by $\sigma_{\tau_{P}} \sim 1 / \sqrt{N_{m c} /\left(2 \tau_{P}\right)}$. The correlated data set is effectively equivalent to an uncorrelated data set consisting of $N_{m c} /\left(2 \tau_{P}\right)$ measurements.

The autocorrelation time associated with an observable $P$ is expected to be governed by the correlation length and near a critical point should behave as

$$
\tau_{P} \sim \xi(T)^{d+z(P)},
$$

with dynamical scaling exponent $z(P)$, which is algorithm and observable dependent. One aim in designing an efficient algorithm is to reduce $z(P)$.

For a critical matrix model with size $N$ we would expect, assuming (40), (47), (48) and (55),

$$
\tau_{P} \sim N^{2+z(P) \frac{2}{d}}
$$

In principle the autocorrelation time for an infinite data set is computed using the series

$$
\tau_{P}=\frac{1}{2} \sum_{n=-\infty}^{\infty} \sum_{\tau=-\infty}^{\infty} \frac{<\left(P_{n}-<P>\right)\left(P_{n+\tau}-<P>\right)>}{<\left(P_{n}-<P>\right)^{2}>}
$$

but in practice the sum $\sum_{\tau=-\infty}^{\infty}$ must obviously be truncated to $\sum_{\tau=-\tau_{0}}^{\tau_{0}}$ with $\tau_{0}$ finite and in general the obtained value for $\tau_{P}$ depends on $\tau_{0}$. Clearly the truncated version of (65) can only be sensitive to autocorrelation times $\lesssim \tau_{0}$ so, if $\tau_{0} \ll N_{m c}, \tau_{p}$ might be underestimated for systems with severe autocorrelation. On the other hand, if $\tau_{0} \sim N_{m c}$, the convergence of (65) becomes very poor. We can not rely only on the above expression to determine $\tau_{P}$.

In our analysis we allow for autocorrelations using the jackknife procedure, see e.g. [27, which computes the uncertainty taking into account the autocorrelation of the data. As a consistency check we compute the autocorrelation using the expression (65), with empirically chosen $\tau_{0}$ and then we compare the result with $\tau_{P}$ as determined by the jackknife procedure. 


\section{References}

[1] T.Banks, W.Fischler, S.Shenker and L.Susskind, "M theory as a matrix model: a conjecture," Phys.Rev. D55 (1997) 5112, [hep-th/9610043].

[2] N.Ishibashi, H.Kawai, Y.Kitazawa, A.Tsuchiya, "A Large N reduced model as superstring," Nucl.Phys. B498 (1997) 467, [hep-th/9612115].

[3] D. Berenstein, J. Maldacena, H. Nastase, "Strings in flat space and pp waves from $N=4$ Super Yang Mills," JHEP 0204 (2002) 013, [hep-th/0202021].

[4] H. Steinacker, "Emergent Geometry and Gravity from Matrix Models: an Introduction," Class. Quant. Grav. 27 (2010) 133001 [arXiv:1003.4134 [hepth]].

[5] R.Myers, "Dielectric branes," JHEP 9912 (1999) 022, [hep-th/9910053].

[6] T. Azuma, S. Bal, K. Nagao and J. Nishimura, "Nonperturbative studies of fuzzy spheres in a matrix model with the Chern-Simons term," JHEP 0405 (2004) 005 [hep-th/0401038].

[7] P. Castro-Villarreal, R. Delgadillo-Blando and B. Ydri, "A Gauge-invariant UV-IR mixing and the corresponding phase transition for U(1) fields on the fuzzy sphere," Nucl. Phys. B 704 (2005) 111 [hep-th/0405201].

[8] T. Azuma, K. Nagao and J. Nishimura, "Perturbative dynamics of fuzzy spheres at large N," JHEP 0506 (2005) 081 [hep-th/0410263].

[9] D. O'Connor and B. Ydri, "Monte Carlo Simulation of a NC Gauge Theory on The Fuzzy Sphere," JHEP 0611 (2006) 016, [hep-lat/0606013].

[10] R. Delgadillo-Blando, D. O'Connor and B. Ydri, "Geometry in transition: A model of emergent geometry," Phys. Rev. Lett. 100 (2008) 201601 [arXiv:0712.3011 [hep-th]].

[11] R. Delgadillo-Blando, D. O'Connor and B. Ydri, "Matrix Models, Gauge Theory and Emergent Geometry," JHEP 0905 (2009) 049 [arXiv:0806.0558 [hep-th]].

[12] R. Delgadillo-Blando and D. O'Connor, "Matrix geometries and Matrix Models," JHEP 1211 (2012) 057 [arXiv:1203.6901 [hep-th]].

[13] J. Madore, “The Fuzzy sphere," Class. Quant. Grav. 9 (1992) 69.

[14] J.Hoppe, "Quantum Theory of A Massless Relativistic Surface and A TwoDimensional Bound State Problem," MIT Ph.D.Thesis, 1982.

[15] M.N. Barber, "Finite Size Scaling" in Phase Transitions and Critical Phenomena, Vol. 8," Edited by C. Domb and J.L. Lebowitz, Academid Press, London 1983, p. 145.

[16] Finite-Size Scaling, Current Physics Sources and Comments, Vol. 2 Edited by John L. Cardy, North-Holland, (1988).

[17] D. E. Berenstein, M. Hanada and S. A. Hartnoll, "Multi-matrix models and emergent geometry," JHEP 0902 (2009) 010 [arXiv:0805.4658 [hep-th]]. 
[18] D. O'Connor and V. G. Filev, "Near commuting multi-matrix models," [arXiv:1212.4818[hep-th]].

[19] V. G. Filev and D. O'Connor, "Multi-matrix models at general coupling," [arXiv:1304.7723 [hep-th]].

[20] A. Pelissetto and E. Vicari, "Critical phenomena and renormalization group theory," Phys. Rept. 368 (2002) 549 [cond-mat/0012164].

[21] B. Widom, "The critical point and scaling theory," Physica 73 (1974) 107.

[22] H. E. Stanley, "Scaling, universality, and renormalization: Three pillars of modern critical phenomena," Rev. Mod. Phys. 71 (1999) S358.

[23] N. Goldenfeld, "Lectures on phase transitions and the renormalization group," Addison-Wesley (1992), Frontiers in physics, 85.

[24] D. O'Connor and C. Nash, "Topological phase transitions and holonomies in the dimer model," J. Phys. A42 (2009) 012002 [arXiv:0809.2960]

[25] J. M. Kosterlitz and D. J. Thouless, "Ordering, metastability and phase transitions in two-dimensional systems," J. Phys. C6 (1973) 1181.

[26] S. Duane, A. D. Kennedy, B. J. Pendleton and D. Roweth, "Hybrid Monte Carlo," Phys. Lett. B195 (1987) 216.

[27] I. Montvay and G. Munster, "Quantum fields on a lattice," (1997), Cambridge University Press.

[28] P. Di Francesco, "2D quantum gravity, matrix models and graph combinatorics," in Application of random matrices in physics. Proceedings, NATO Advanced Study Institute, Les Houches, France, June 6-25, 2004 [math$\mathrm{ph} / 0406013]$. 